\title{
Contextual Learning Analytics Apps to Create Awareness in Blended Inquiry Learning
}

\author{
Andrii Vozniuk, María Jesús Rodríguez-Triana, Adrian Holzer, Sten Govaerts, David Sandoz, Denis Gillet \\ School of Engineering, EPFL, Station 9, CH-1015 Lausanne, Switzerland \\ \{firstname. lastname\} depfl.ch
}

\begin{abstract}
Blended inquiry learning relying on online labs to enhance classroom activities is considered as a promising approach to increase the skills and the interest of students in science, technology, engineering, and mathematics. In such a framework where students combine face-to-face interaction with peers or teachers and online interaction with remote or virtual labs, adequate guidance should be provided. Such a task is facilitated if teachers are made aware of the progresses and the difficulties of their students. Learning analytics based on the analysis of the student online interaction traces and their visualization is an adequate mean. A set of contextual learning analytics apps which provide the teacher with learning-specific information is introduced in this paper. A requirement analysis gathered at a summer school with 32 teachers, the design and implementation of three contextual learning analytics apps, the main outcomes from a case study, as well as an outlook on future research avenues are detailed.
\end{abstract}

\section{INTRODUCTION}

Blended inquiry learning relying on online labs to enhance classroom activities is considered as a promising approach to increase the skills and the interest of students in science, technology, engineering, and mathematics (STEM). For instance, the Go-Lab European project ${ }^{1}$ aims at promoting inquiry-based learning using online labs through its web portal [1], [2].

To enable teachers to orchestrate a blended learning session and to provide adequate guidance to their students, it is critical that they are aware of their progresses and difficulties. Especially, in a blended learning activity, it is often challenging for the teacher to be aware of what the students are doing and how they are doing it in order to intervene when necessary. This task is not trivial for both small and large classes, as in such a blended learning framework part of the work is done online. This however makes possible to record student activity traces and to provide the teacher with dedicated analytics to improve awareness. To represent important information about learners, Duval et al. propose to use learning analytics (LA) dashboards [3]. According to Stephen Few [4] such a dashboard is a visual display of the most important information needed to achieve one or more objectives, consolidated and arranged on a single screen so the information can be monitored at a glance.

In this paper our aim is to build learning analytics apps and dashboard in the context of blended learning sessions and verify if such tools are useful for the teachers to improve their awareness. The paper is structured as follows. Section 2 introduces Graasp, an example of a learning environment that can be used to conduct blended learning sessions and where our analytics tools are integrated. Section 3 presents the requirements of teachers for awareness tools. Section 4

\footnotetext{
${ }^{1}$ Go-Lab, http://www.go-lab-project.eu/
}

discusses related work and point to shortcomings. Section 5 introduces the concept of contextual learning analytics apps and presents three such apps that fulfill the requirements elicited in Section 3. Section 6 presents a case study of the usage of the apps in a real life blended session. Finally, Section 7 wraps up with a conclusion.

\section{GRAASP}

In the Go-Lab project teachers use the Graasp platform to conduct blended learning activities. Graasp ${ }^{2}$ is a social media platform designed to support collaboration, learning and knowledge management [5]. More broadly, Graasp supports many types of collaboration and is especially well suited for agile and decentralised structures such as schools, universities and non-governmental organisations (NGOs) [6]. A space is the central concept in Graasp. It encapsulates the context of a collaboration. A space can be compared to a folder in a file system with members having permissions defined by their role (as owner, editor or viewer). In inquiry-based learning, the learner is expected to go through a set of phases, e.g., Orientation, Conceptualisation, Investigation, Conclusion, Discussion, sometimes sequentially, but also moving back and forth between phases [7]. Graasp provides a special type of space, called Inquiry Learning Space (ILS) with the aforementioned inquiry learning phases as sub-spaces of the ILS embedding relevant content (e.g., PDF's, instructions \& videos), apps (e.g., concepts maps, chat \& drawing tools) and labs (e.g., chemistry simulations and remote hardware such as a telescope) [8].

Graasp provides an authoring view and a standalone view. The former is used by the teachers to assemble an ILS and the latter is accessed by students through a secret URL that the teacher shares with them. Figure 1 shows an example of the standalone view. Each phase of the inquiry cycle is located in a different tab. In this particular case, the student, named David, is currently in the Orientation phase of the Gears ILS where several examples of gears are presented.

\section{RELATED WORK}

The research literature provides a number of examples of learning analytics tools developed to improve awareness of teachers in a classroom [9]. As a general approach, Segedy et al. [10] propose a model for integrating an intelligent learning environment into a classroom, where the environment records and extracts information about student behaviour in the class, informs the teachers about this behaviour and the teachers use their pedagogical expertise for appropriate reaction [10]. Following this general approach, below we review several 


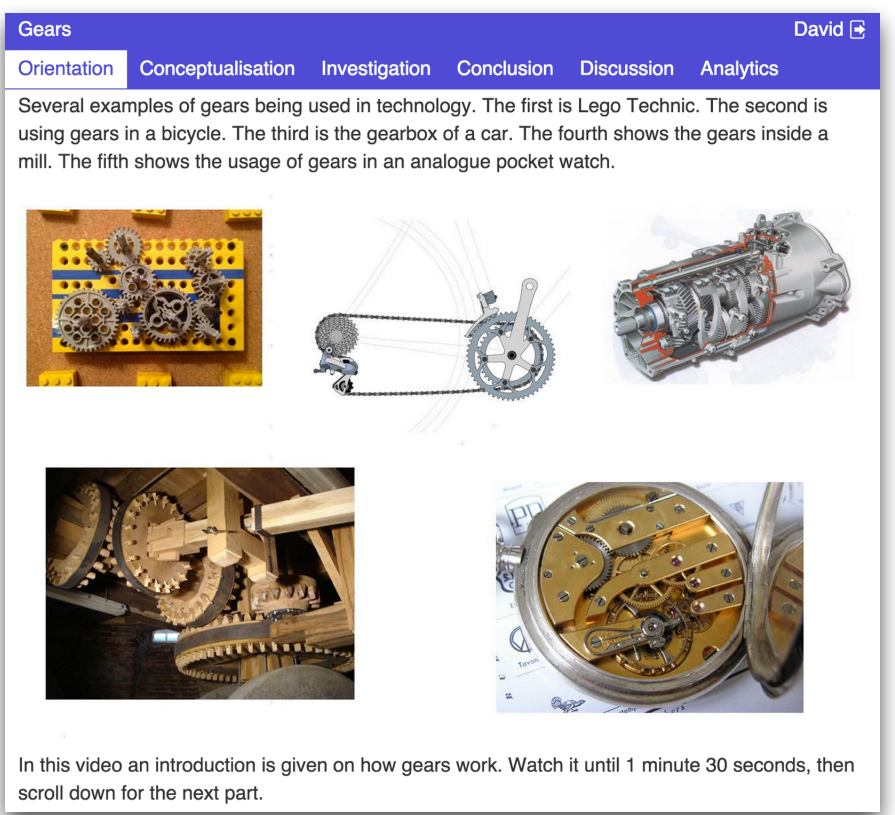

Fig. 1. Example of the standalone view of an ILS

specific examples of awareness tools being deployed and used in different learning contexts.

Maldonado et al. [11] present a set of real-time visualisations that help teachers to be aware of the interaction patterns in tabletop-based group work. The visualisations are based on tabletop touch data and verbal data recorded by microphones. The authors suggest to visualise presented interaction on two levels: (1) class level, aggregating data from interactions around several tabletops in the classroom (useful to identify anomalies during a classroom session) and (2) detailed group level helping to detect possible anomalies in a single group (useful for after-class analysis). A user study showed that such visualisation tools are useful for the teacher and help her to identify the groups and individual students that require attention and intervene when necessary. The study also demonstrated that the teachers were able to detect the main anomalies in group interactions based on the class level while the detailed group level was used to confirm the assumed issues.

Charleer et al. propose to employ a learning analytics dashboard as a course-long awareness tool for teachers and students [12]. During the course, the students interact using twitter and blogging platforms and their activity traces are being recorded. A set of badges is used as an indicator of the student progress towards the course goals and the level of student activity in the course. The dashboard allows to track the progress of individual students and compare the progress among the students. Since teachers can be overwhelmed by manually observing each of the student activities, such a dashboard can help them to remain aware of the student progress just by checking if the students have received all expected badges at a given time. If a more detailed view of the progress is needed, teachers can look into individual actions of the students.

From the implementation point of view, the considered tools rely on custom data collection and visualisation in- frastructures. Another option of building the tools could be to use general-purpose web analytics platforms (e.g. Google Analytics $^{3}$ or KISSMetrics ${ }^{4}$ ). They are easy to deploy and maintain, but they come with a number of shortcomings. First of all, storing student activity traces on third-party services raises privacy concerns. Some platforms such as Google Analytics explicitly forbid in their service license agreement to send to the platform any data explicitly revealing the user identity. Additionally, web analytics tools usually aggregate and display the data for the whole web platform. When no identity information is stored, it is not possible to track performance of individual students. In the case of the Go-lab portal, teachers are more interested to see analytics for their individual ILSs with the possibility of viewing data about their individual students. Retrieving such information from web analytics tools is not always possible and when possible often requires writing custom queries. Finally, web analytics tools usually do not provide expressive semantics for the learning context, i.e. they are able to identify web page visits made by students, but not if a student has changed an ILS phase or submitted an assignment.

\section{AWARENESS NEEDS}

To understand the awareness information needs of the teachers, we interviewed 32 teachers during the 2014 GoLab Summer School asking them which kind of learning analytics application would be helpful during their blended learning sessions. Teachers were asked to what extend they agreed with the following statement: "I would use this app in class". In addition, they were asked about the relevance of having an overview screen (dashboard). We used 7-point Likert scale ranging from -3 (strongly disagree) to +3 (strongly agree) to collect the responses. Afterwards, we calculated the mode of the score per app; the box plot visualising the survey data is presented in Figure 2. According to the results, the apps that received the highest score are: 1) submission by student, 2) average time per phase, 3) connected students and 4) connected students per phase. The figure also demonstrates that the teachers were eager to have and use a single overview screen (dashboard) containing several apps at a time.

\section{The Contextual Learning Analytics Apps}

In this section we first introduce contextual learning analytics (LA) apps that aim to improve the awareness of teachers in blended inquiry sessions. We continue by discussing the technology behind the apps; afterwards we present three examples of such apps and discuss a typical scenario in which these apps can be used in the classroom.

We call the apps "contextual" since such apps can be embedded into the context where the students and the teacher work, understand the context and visualise the data present in this specific context. Since the apps are located in the context where the teacher works (in our case ILS), she does not need to leave that context in order to see analytics, thus avoiding context switching. We provide the teacher with a set of widgets that can be used in a specific ILS context. When the teacher wants to view several visualisations at a time,

\footnotetext{
${ }^{3} \mathrm{http}: / /$ www.google.com/analytics/

${ }^{4}$ https://www.kissmetrics.com/
} 


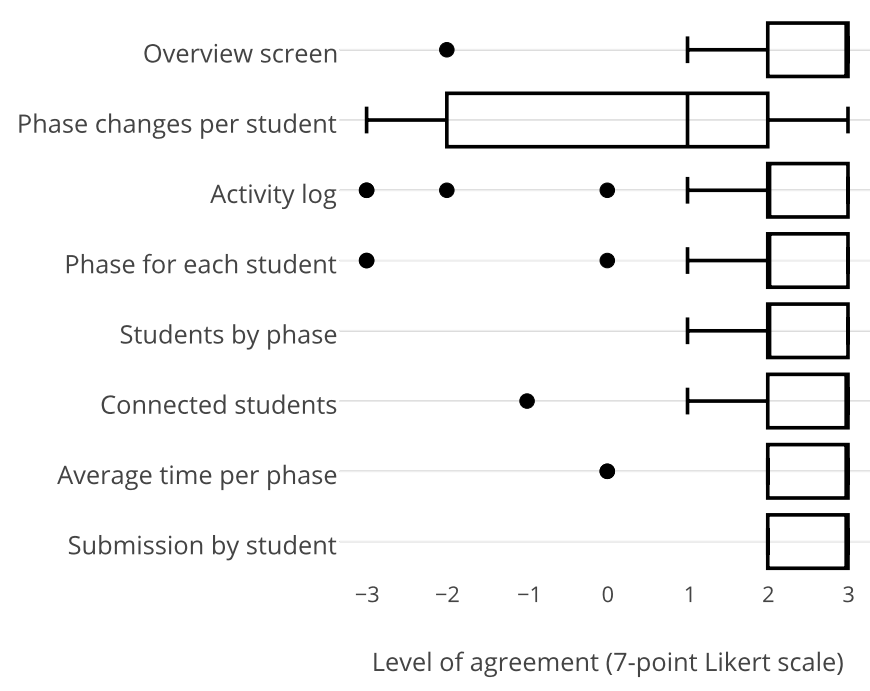

Fig. 2. Results of the awareness needs survey.

Graasp allows her to organise several LA apps on a single analytics dashboard. The ILS analytics dashboard shows a set of indicators in individual apps: students in phases, time spent, etc. The purpose of the apps is to present real-time activity traces recorded by a web platform in an interactive dashboard improving teacher awareness.

Each user (student and teacher) using the Graasp platform produces a stream of activities reflecting their interaction with the platform. We use the ActivityStreams specification ${ }^{5}$ to represent each of the actions. The actions are created and retrieved via the OpenSocial API. Each time a new activity occurs in the ILS, it is saved in Graasp via the OpenSocial API and then Graasp emits it through a WebSocket to the gadget. From technology point of view, our LA apps [13] are small OpenSocial gadgets [14] built with HTML, CSS and JavaScript. When the app is loaded for the first time it gets the data about the structure of the ILS and all the past actions via the OpenSocial API. Afterwards, the app receives realtime updates about the new activities using the WebSocket protocol ${ }^{6}$. Since the contextual apps rely on well-specified OpenSocial APIs to retrieve the data, they are portable and interoperable across the ILSs and often across the learning management systems implementing these APIs [13].

To encourage the sharing and reuse inside of the Go-Lab project, the Golabz ${ }^{7}$ lab repository was developed. It can be used to publish new apps and search for existing apps. Since awareness needs of the teachers can differ in different contexts (ILSs), they can use existing apps to assemble dashboards reflecting their specific needs in each of the contexts. Below we discuss in more detail three contextual analytics apps implemented to address the awareness needs identified in Section IV. Along with the apps, we present a typical scenario in which the apps can be used in a blended session.

\footnotetext{
${ }^{5}$ Activity Streams Specification: http://activitystrea.ms/specs/json/1.0/

${ }^{6}$ What is WebSocket?, https://www.websocket.org/

${ }^{7}$ Golabz Repository: http://www.golabz.eu/
}

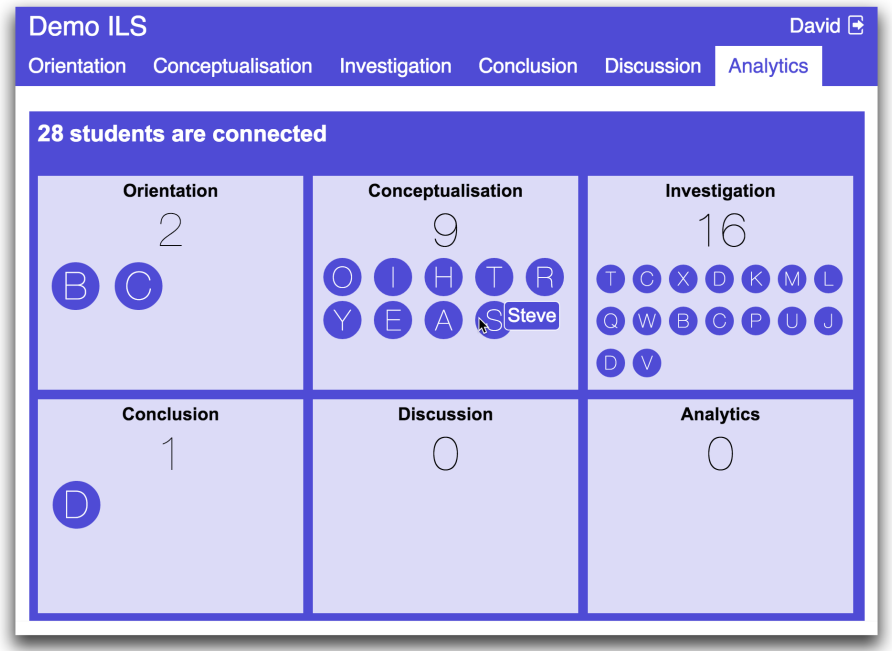

Fig. 3. The Active Users app displaying the students active in the ILS.

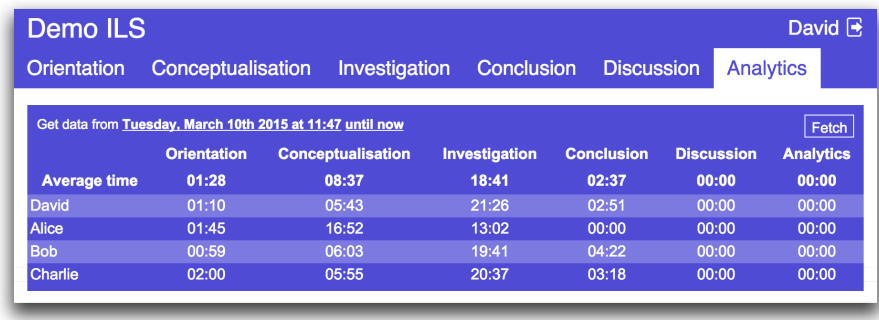

Fig. 4. The Time Spent displaying the time spent by students in the ILS.

\section{A. Active Users App}

Figure 3 shows the Demo ILS with the Active Users app displaying the students currently active in each phase of the ILS. It is possible to see that the majority of the students are in the Investigation and the Conceptualisation phases (16 and 9 respectively), there are 2 under-performing students active in the Orientation phase and 1 outperforming student in the Conclusion phase. The app updates in real time as students switch the phases, so the teacher knows where the students are working right now.

\section{B. Time Spent App}

Figure 4 shows the Demo ILS with the Time Spent app displaying individual and average time spent by students in each ILS phase. When the student is active in one specific space, the teacher is able to see the timer running for this student in that space in real time. Additionally, the average time spent by students in each phase is given so it is possible to compare individual performance of students to average performance of the group. There is also the possibility to analyse a specific period of time, for instance if the teacher wants to perform a post-analysis of the session.

\section{Submitted Reports App}

Figure 5 shows the Demo ILS with the Submitted Reports app displaying the files submitted by students in the ILS and the time when it was done. It is updated in real time as well. Therefore, every time a new report file is uploaded in any phase of the ILS, a new line appears in the interface. 


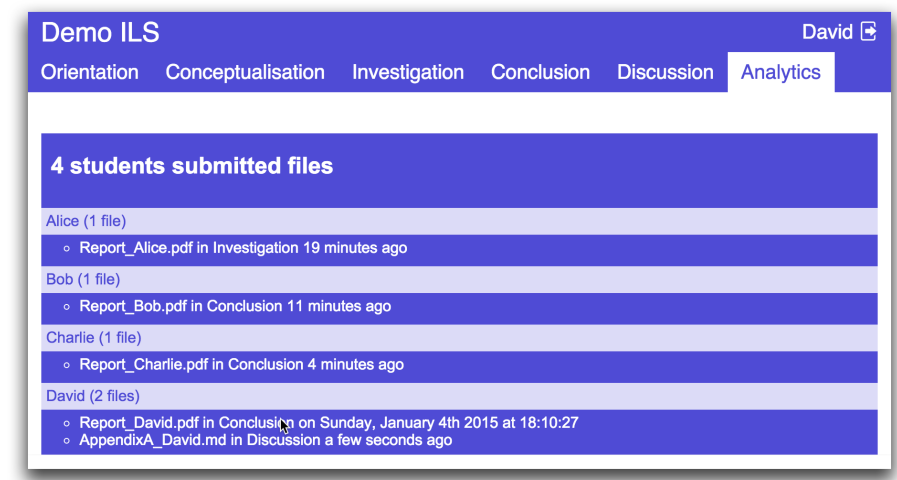

Fig. 5. The Submitted Reports app displaying the submitted reports.

\section{Scenario}

Below we describe a scenario how the apps can be used in a blended learning session.

Before the session the teacher adds the Active Users, Time Spent app Submitted Reports into the ILS.

In the beginning of the session she looks at the Active Users app and is able to see the students appear in the Orientation phase as they open the ILS on their computers. During the session, as the students complete the Orientation phase, they switch to the Conceptualisation phase, and the teacher is able to see it in the Active Users app shown in the example in Figure 3. With this app the teacher spots the underperforming students that are still in the Orientation phase. The teacher then goes to the students and helps them accordingly. The teacher is using the Time Spent app and observes that almost all the students have spent between 4 and 6 minutes in the Conceptualisation phase as expected. In the end of the session the students submit a report in the Conclusion phase. As the submissions happen, the reports appear in the Submitted Reports app, which gives an overview of who has submitted as demonstrated in Figure 5. There are few minutes left till the end of the session and the app indicated that 26 students have already submitted files. The teacher identifies who are the 2 students who still need to submit their files and goes to them directly to see if there is an issue.

After the session, the teacher uses the Time Spent app to evaluate how much time was spent on average by students in each phase. She notices that in average the students have spent almost 19 minutes in the Investigation phase, which is too much. This led the students to rush through the Discussion phase. She will therefore make the necessary changes in the description of the Investigation phase so the next time students will complete it in less time.

\section{Preliminary EVAlUation}

We have conducted a preliminary evaluation of the usability and the usefulness of the implemented apps for teachers. Two case studies using the Active Users, the Time Spent and the Submitted Reports apps were carried out in Geneva between January and March 2015. The first study took place at the Ecole de Commerce Nicolas-Bouvier involving 1 teacher (T1) and 11 students (between 18 and 20 years old). The second study was conducted at the College Sismondi where 1 teacher (T2) and 17 students (between 15 and 16 years old)

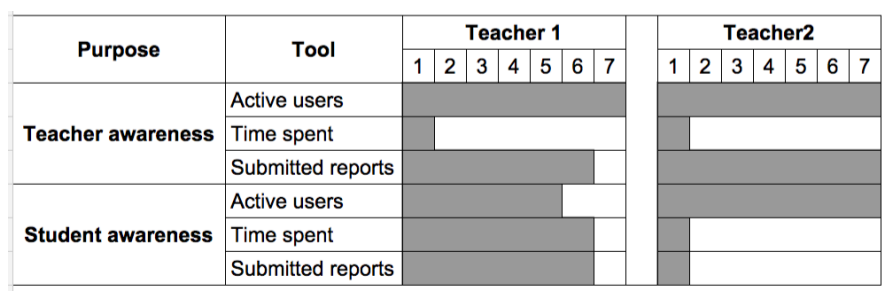

Fig. 6. Relevance of the apps according to the teacher interviews.

participated in the experiment. The aim of these studies was to answer the following research questions: RQ1. Do such contextual real-time visualisations improve teacher's awareness? RQ2. Are the tools understandable and easy to use?

To answer these questions we used three data sources: first, we interviewed the teachers before the experiments to evaluate whether the information provided by the apps could be relevant for them and with which purpose; then, one researcher attended the classroom to observe how the apps were used during the lessons and collected students comments about the apps; finally, we gathered additional remarks from the teachers in a second interview once they had used the apps.

In the first interview, the teachers were asked about the extent to which they use awareness apps for teachers and students. The main outcomes of the interview are summarised in Figure 6, showing the teachers' point of view using a 7point Likert scale ranging from 1 (strongly disagree) to 7 (strongly agree). Dealing with the Active Users app, both T1 and T2 agreed on the usefulness of the app for awareness of the learning progress for teachers and students. The Time Spent app was not seen as significantly useful for awareness. However, both teachers agreed that it could be relevant for better understanding the students' progress. Concerning the potential convenience for students, both teachers came up to the conclusion that the app could help learners to be aware of their peers' progress. Regarding the Submitted Reports app, the teachers considered that they would use it for their own awareness but they diverged about the benefits that the app could bring to the students.

In both studies, the teachers designed an ILS to be used by the students during a 90-minute session. In the first study, the students were allowed to work individually or in groups, and teacher was walking around and answering the questions that emerged during the learning activity. Since the teacher was not nearby her computer, she decided to display the apps using the projector, so teachers and students could see the visualisations as presented in Figure 7. After answering the questions of the students, the teacher had a look at the student distribution across inquiry phases and used that information to choose the next group to visit. At the same time, the students periodically observed the apps to compare their own progress with that of their peers.

In the second study, the students worked in groups of 2 to 3 people sharing one computer, while the teacher controlled the situation from his desk, going to the students just when he had doubts.T2 used mainly the Active Users app to monitor whether the students were using the ILS or not, and to be aware of the current phase they were working on. Since, in this case, the information was not shown to the students, at the 


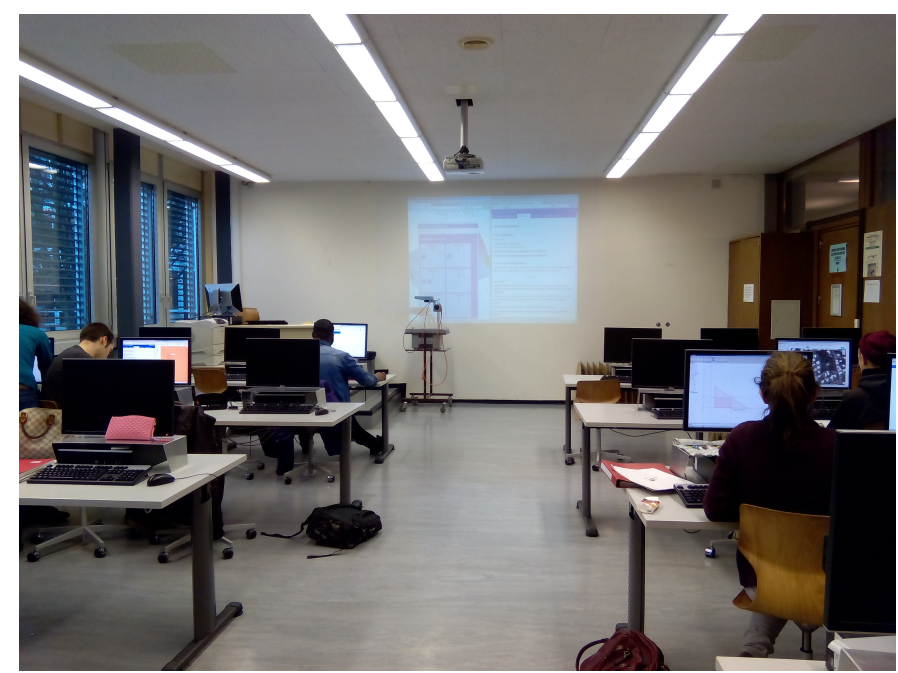

Fig. 7. Use of the Active Users app for teacher and student awareness.

end of the lesson they were asked about whether they would like to have access to the apps or not. The responses were heterogeneous: 7 students did not have a clear preference, 4 were highly interested in the apps, while another 6 students were reluctant. For those who were interested in the apps, the main benefit was to have a reference about the progress. The main disadvantages identified by the students were 1) the distraction the apps may cause and 2) the stress caused by being compared among themselves. Nevertheless, they did not pose any concern about the teacher accessing the information.

According to the feedback provided by the teachers, in general terms, it was straightforward to understand the visualisation provided by the apps. Just one aspect required additional explanation for the teachers in relation to the Active Users app: the colours used to show the time spent. For T1, the only inconvenience that she detected was related to the way the users were represented. Since she was not near her computer, the mapping between the student initials (used in the avatar) and the nicknames was not obvious. In addition, the different colours employed to show the time spent were not distinguished when projected on the wall. Nevertheless, both issues could be simply solved by using a legend explaining the colours and the correspondence between initial and nickname, and choosing different colours, shapes or effects. Regarding the Time Spent app, both teachers agreed that it can be more useful for post-session reflection, especially since during the session normally they do not have enough time for these details.

In summary, real-time awareness tools were well received by the teachers both in terms of usability and applicability. They stated that the tools helped them to monitor the progress of the students in the classroom but they could be also useful in order to have evidence of the work done at home.

\section{CONCLUSION \& FUTURE WORK}

This paper presented an approach to recording and visualising with help of contextual learning analytics apps activities performed by students in a blended learning session. We have designed and built three sample apps to address the identified main awareness needs of the Go-Lab teachers. These apps target common scenario where each of the students does their individual work. As part of the future work, it is possible to develop apps dedicated to providing awareness in collaborative work during blended sessions.

When building the apps, we targeted the teacher awareness needs, but during the evaluation the students expressed such needs as well, for instance to make them aware of their progress compared to the progress of others. In the future we want to identify the awareness needs of students and build apps that improve student awareness. However, during the evaluation we also noticed that some students were not happy about others seeing the progress, we think that the questions of students data privacy is worth investigating.

\section{ACKNOWLEDGMENT}

This research is partially funded by the European Union in the context of the Go-Lab project (Grant Agreement no. 317601) under the Information and Communication Technologies (ICT) theme of the 7th Framework Programme for R\&D (FP7). This document does not represent the opinion of the European Union, and the European Union is not responsible for any use that might be made of its content.

\section{REFERENCES}

[1] T. de Jong, S. Sotirou, and D. Gillet, "Innovations in STEM education: The Go-Lab federation of online labs," Smart Learning Environments, vol. 1, no. 3, 2014.

[2] S. Govaerts, Y. Cao, A. Vozniuk, A. C. Holzer, D. Garbi Zutin, E. San Cristbal Ruiz, L. Bollen, S. Manske, N. Faltin, C. Salzmann, E. Tsourlidaki, and D. Gillet, "Towards an Online Lab Portal for Inquiry-based STEM Learning at School," in ICWL, 2013.

[3] E. Duval, "Attention please! learning analytics for visualization and recommendation," in Proceedings of LAK11: 1st International Conference on Learning Analytics and Knowledge, 2011, pp. 9-17.

[4] S. Few, Information dashboard design. O'Reilly, 2006.

[5] D. Gillet and E. Bogdanov, "Contextual spaces as agile personal learning environments or informal knowledge management solutions," in ITHET, 2013.

[6] A. Vozniuk, A. Holzer, S. Govaerts, J. Mazuze, and D. Gillet, "Graspeo: a Social Media Platform for Knowledge Management in NGOs," in ICTD, 2015.

[7] T. de Jong, "D1.1: Go-Lab learning spaces specification," GoLab European Project, Tech. Rep., 2013. [Online]. Available: http://www.go-lab-project.eu/deliverables

[8] M. J. Rodriguez Triana, S. Govaerts, W. Halimi, A. C. Holzer, C. Salzmann, A. Vozniuk, T. de Jong, S. Sotirou, and D. Gillet, "Rich Open Educational Resources for Personal and Inquiry Learning. agile Creation, Sharing and Reuse in Educational Social Media Platforms," in International Conference on Web \& Open Access to Learning, 2014.

[9] K. Verbert, S. Govaerts, E. Duval, J. L. Santos, F. Van Assche, G. Parra, and J. Klerkx, "Learning dashboards: an overview and future research opportunities," Personal and Ubiquitous Computing, vol. 18, 2014.

[10] J. Segedy, B. Sulcer, and G. Biswas, "Are iles ready for the classroom? bringing teachers into the feedback loop," in Intelligent tutoring systems. Springer, 2010, pp. 405-407.

[11] R. M. Maldonado, J. Kay, K. Yacef, and B. Schwendimann, "An interactive teachers dashboard for monitoring groups in a multi-tabletop learning environment," in Intelligent Tutoring Systems. Springer, 2012.

[12] S. Charleer, J. L. Santos, J. Klerkx, and E. Duval, "Improving teacher awareness through activity, badge and content visualizations," in New Horizons in Web Based Learning. S Springer, 2014.

[13] A. Vozniuk, S. Govaerts, and D. Gillet, "Towards portable learning analytics dashboards," in ICALT, 2013.

[14] M. Häsel, "Opensocial: an enabler for social applications on the web," Communications of the ACM, vol. 54, no. 1, pp. 139-144, 2011. 